\title{
Scattering polarization in strong chromospheric lines
}

\section{Influence of the temperature curve on the Call $\mathrm{K}$ line}

\author{
R. Holzreuter ${ }^{1}$ and J. O. Stenflo ${ }^{1,2}$ \\ 1 Institute of Astronomy, ETH Zentrum, 8092 Zurich, Switzerland \\ e-mail: [holzreuter;stenflo] @astro.phys.ethz.ch \\ 2 Faculty of Mathematics \& Science, University of Zurich, 8057 Zurich, Switzerland
}

Received 14 November 2006 / Accepted 23 January 2007

\begin{abstract}
In a previous paper we analyzed the polarization profile of the Ca II K line near the limb at $\mu=0.1$. We now extend this work to model the center-to-limb variation of the linear polarization of the Ca II $\mathrm{K}$ line and compare it with calculated spectra based on standard 1D model atmospheres. Our previous two-component approach with a hot and a cool atmospheric component is re-examined. We confirm our previous result that no single model is able to explain the observations. While self-consistent single atmosphere solutions may fit the polarization spectra, they fail to simultaneously fit the corresponding intensity spectra or the polarization spectra at other heliocentric angles. The mixing of a cool and a hot component is however a good approach for all disk positions, although the optimum mixing ratio varies strongly with heliocentric angle. As we approach the limb the hot component gains in importance, which is consistent with the scenario of diverging magnetic canopies overlying a cool atmosphere.
\end{abstract}

Key words. line: formation - polarization - radiative transfer - scattering - Sun: atmosphere - Sun: chromosphere

\section{Introduction}

In our previous work (Holzreuter et al. 2005, 2006, henceforth Papers Ia and Ib), we demonstrated the strong dependence of the polarization of chromospheric lines on the temperature profile of the atmosphere. In particular, the very strong Ca II $\mathrm{K}$ line is a good tool to diagnose the temperature around the classical temperature minimum. The enhancement of the polarization at $\mathrm{K} 1$ and the weakness of the polarization dip at K2 in comparison with theory (Stenflo 1980) and calculations (Auer et al. 1980; Saliba 1985, and Paper Ib) led us to the conclusion that no established atmospheric model with the classical temperature rise (Fontenla et al. 1993) could explain the observations. Only when we use a two-component model by adding a substantial part of a much colder model FAL-X, used by Avrett (1995, his model $\left.M_{\mathrm{CO}}\right)$ to explain molecular CO lines, the calculations fit the observations well.

The present work is based on a series of observations in the $\mathrm{Ca}$ II $\mathrm{K}$ line at different heliocentric angles, which was obtained at the Mc Math-Pierce telescope of NSO (Kitt Peak) in March 2005. The data set demonstrates the outstanding potential of ZIMPOL (Povel 1995; Stenflo \& Keller 1997; Gandorfer et al. 2004) for measuring polarization in strong lines. It was possible to measure the full Stokes vector for every heliocentric angle between $\mu=\cos \theta=0.1$ and 1.0 in steps of 0.1 , although the polarization signals at larger $\mu$ get very small due to geometric factors. All profiles have a high quality and show very similar features, even at disk positions with $\mu>0.7$.

In Paper Ib the comparison with theory was based on one single observation at the limb (Gandorfer 2002). The new data set allows a re-examination of the hypothesis of temperature bifurcation, which is assumed to take place in the lower chromosphere. It enables us to impose much stronger constraints on the modeling: Each model has to explain all the observations simultaneously. Different wavelength positions at different observing angles are now indirectly linked by the common formation height.

\section{Data acquisition and line polarization modeling}

The data set used in the present work was obtained with the ZIMPOL UV-sensitive camera system (Gandorfer et al. 2004) during three days at the Mc Math-Pierce facility of NSO (Kitt Peak) in March 2005 (Stenflo 2006). The slit covered approximately 80 arc seconds and was positioned in the southern hemisphere and oriented perpendicular to the radius vector from disk center (i.e. parallel to the nearest limb). The exposure time for a single measurement was about five minutes. At each heliographic position, two full Stokes vector spectra covering $5.6 \AA$ each with an overlap of about $0.8 \AA$ were recorded. The overlap is used in the data reduction to adjust the relative position of the zero points of the polarization scales for the two spectra.

One fundamental aspect of the data reduction is the removal of instrumental polarization from the observed spectra. The general procedure indicated in a previous publication (Stenflo et al. 2001) has, over the last several years, been refined and applied to the various ZIMPOL observations that have since been obtained, but as a description of the method has not yet been published (apart from some discussion of cross talk removal when observing with a filter system, cf. Holzreuter 2003; Stenflo et al. 2002), we describe the method here in some detail for completeness.

The intrinsic polarizations $Q / I, U / I$, and $V / I$ are in our observations very small, $\lesssim 1 \%$. Substantially larger, spurious polarizations are created by telescope cross talk from Stokes $I$ into the other Stokes parameters. Ideally the corresponding coefficients for cross talk from Stokes $I$ should be spectrally flat and produce a spectrally flat offset of the zero point of the polarization 
scale when $Q, U$, and $V$ are divided by $I$ to form the fractional polarizations. The removal of this cross talk then reduces to the task of finding the correct zero point.

If, however, the spurious polarization created by cross talk from Stokes $I$ is too large, as it is with the large, time-varying instrumental polarization of the McMath telescope, then effects of deviations from linearity in the detector response will come into play and generate fictitious polarization signatures, which would not disappear by any zero-line shift. To reduce such effects to below the $10^{-5}$ level in the polarization, it is necessary to use a servo-controlled plane parallel glass plate as a polarization compensator during the observations, which in real time minimizes both Stokes $Q$ and $U$ (as averaged over the selected spectral window).

The removal of the instrumental polarization in the data analysis therefore effectively means that we need to invert the Mueller matrix that represents the combination of the telescope and the compensating plate. This is basically done in two stages, first through the removal of the cross talk between the $Q, U$, and $V$ parameters, and then by finding and applying the proper zero-line shifts to $Q / I, U / I$, and $V / I$.

Cross talk between the $Q, U$, and $V$ parameters can be seen as being produced by phase shifts (retardations) caused by each optical element or each reflection. The polarization effect of such phase shifts by wave plates or equivalent elements can be described in terms of rotations of the Stokes vector in the 3D Poincaré space that is spanned by the Stokes parameters $Q, U$, and $V$. The length $\left.\sqrt{(} Q^{2}+U^{2}+V^{2}\right)$ of the polarization vector is not affected, only the location of its end point on the Poincare sphere. The axis of rotation depends on the orientation of the optical axis of the corresponding equivalent wave plate. When there is a succession of wave plates with different orientations of their axes, the effect is obtained through a succession of corresponding rotations in Poincaré space.

The net end effect is that the $(Q, U, V)^{\mathrm{T}}$ vector is reoriented while its amplitude remains unchanged. As this reorientation does not depend on the particular $(Q, U, V)^{\mathrm{T}}$ vector that we choose to look at, but is a property of the optical components independent of the polarization state of the light beam that traverses the system (linear optics), we can conceptually view the rotations in the inverse way: we may let the polarization vectors $(Q, U, V)^{\mathrm{T}}$ (which vary greatly with wavelength and position on the Sun) all remain fixed, while we instead rotate the Poincare sphere (with its attached $Q, U$, and $V$ coordinate axes) in the opposite direction. These rotations simultaneously apply to all the Stokes vectors.

Removal of this cross talk between the Stokes parameters is achieved if we can apply rotation matrices that bring the Poincaré sphere back to its original orientation. There are many ways to achieve this (like there are many choices for a sequence of rotations to solve Rubik's cube problem), one does not at all need to know in what sequence the Poincaré sphere got oriented in the first place in its particular way. The unscrambling of the problem can be done in an entirely different way.

The stepwise unscrambling procedure is to start by picking a pair of Stokes parameters, e.g. $Q$ and $V$, and remove the cross talk between them as well as possible. This procedure corresponds to a rotation of the Poincaré sphere around the $U$ axis. If the angle of rotation is $\alpha_{Q V}$, the Stokes parameters transform as $Q^{\prime}=Q \cos \alpha_{Q V}+V \sin \alpha_{Q V}$ and $V^{\prime}=-Q \sin \alpha_{Q V}+V \cos \alpha_{Q V}$. The rotation, defined by parameter $\alpha_{Q V}$, automatically takes care of the cross talk in both directions, both $Q \rightarrow V$ and $V \rightarrow Q$. It would be incorrect to consider these two directions as separate cases.
Any arbitrary instrumental polarization can then be removed, i.e., the spurious orientation of the Poincaré sphere can be unscrambled, through a succession of three rotations around the three fixed coordinate axes, by a proper choice of the three angles $\alpha_{Q V}, \alpha_{U V}$, and $\alpha_{Q U}$. As the optimum angles may not be found with the first attempt, the removal can be done iteratively: one first makes an approximate selection of the three angles and thereby eliminates the instrumental cross talk to first order. Using the so corrected Stokes data as a new starting point, one does fine tuning by selecting new, much smaller rotation angles, which brings the correction to the next order of precision, and so on. In each step and thus throughout the whole procedure, the amplitude of the polarization vector remains invariant, only its orientation is modified. The rotations can be applied in any order, although faster convergence is obtained if the cross talks having the larger rotation angles are applied before those with smaller angles. The application is done interactively.

The main issue then boils down to the question how the angles $\alpha$ are chosen. For this we use the polarization signatures that reflect the very distinct and well known symmetry properties of the Zeeman and Hanle effects. For a first order cross talk removal one uses the two criteria that (i) the anti-symmetric profile shapes that are characteristic of the longitudinal Zeeman effect should not be visible in the $Q$ and $U$ spectra, only in Stokes $V$, and (ii) the scattering polarization in the wings of a scattering line should only be seen in $Q$ but not in $U$ (assuming that the Stokes system is chosen such that the $Q$ direction is parallel to the nearest solar limb). Criterion (i) fixes the values of $\alpha_{Q V}$ and $\alpha_{U V}$, while criterion (ii) fixes the angle $\alpha_{Q U}$.

Usually the application of these two criteria leads to immediate convergence, such that no cross talk remains visible in the Stokes spectra. However, additional checks and secondary criteria may be applied to improve the correction when needed. If for instance traces of scattering polarization signatures are seen in Stokes $V$, then a correction to the $\alpha_{Q V}$ angle needs to be applied.

All the criteria thus used refer to the polarized profile shapes, but do not address the zero point shifts of the polarization scale. Incorrect polarization zero levels do not affect the procedure for the elimination of the cross talk between $Q, U$, and $V$. The rotations in Poincaré space will affect the magnitudes of these zero point shifts, but no spurious polarization features will be generated from the zero offsets by the rotations. Therefore the zeroline corrections can be done after all the other cross talks have been eliminated.

For Stokes $U$ and $V$ the correct zero points are easily found by the requirement that the polarization in the continuum (or quasi-continuum in the case of the extended wings of the $\mathrm{Ca} \mathrm{K}$ line) should be zero. The only remaining parameter is then the zero point for the $Q$ spectrum. For its determination we make use of the numerous depolarizing blend lines in the extended wings of the $\mathrm{Ca} \mathrm{K}$ line. Since there is much experience in the application of this technique in previous spectral surveys (e.g. Stenflo et al. 1983; Gandorfer 2002), and the behavior of the depolarizing lines has been thoroughly explored by theory (Fluri $\&$ Stenflo 2003), the $Q$ zero point can be obtained this way with great confidence.

Our method to calculate the synthetic spectra is described in Papers Ia and Ib. The calculations presented here are made with the same atomic and atmospheric parameters, except that we extend the set of atmospheric standard models by the FAL-A and FAL-F model of Fontenla et al. (1993). All calculations are performed without Hanle depolarization (i.e. with $B=0$ ). 

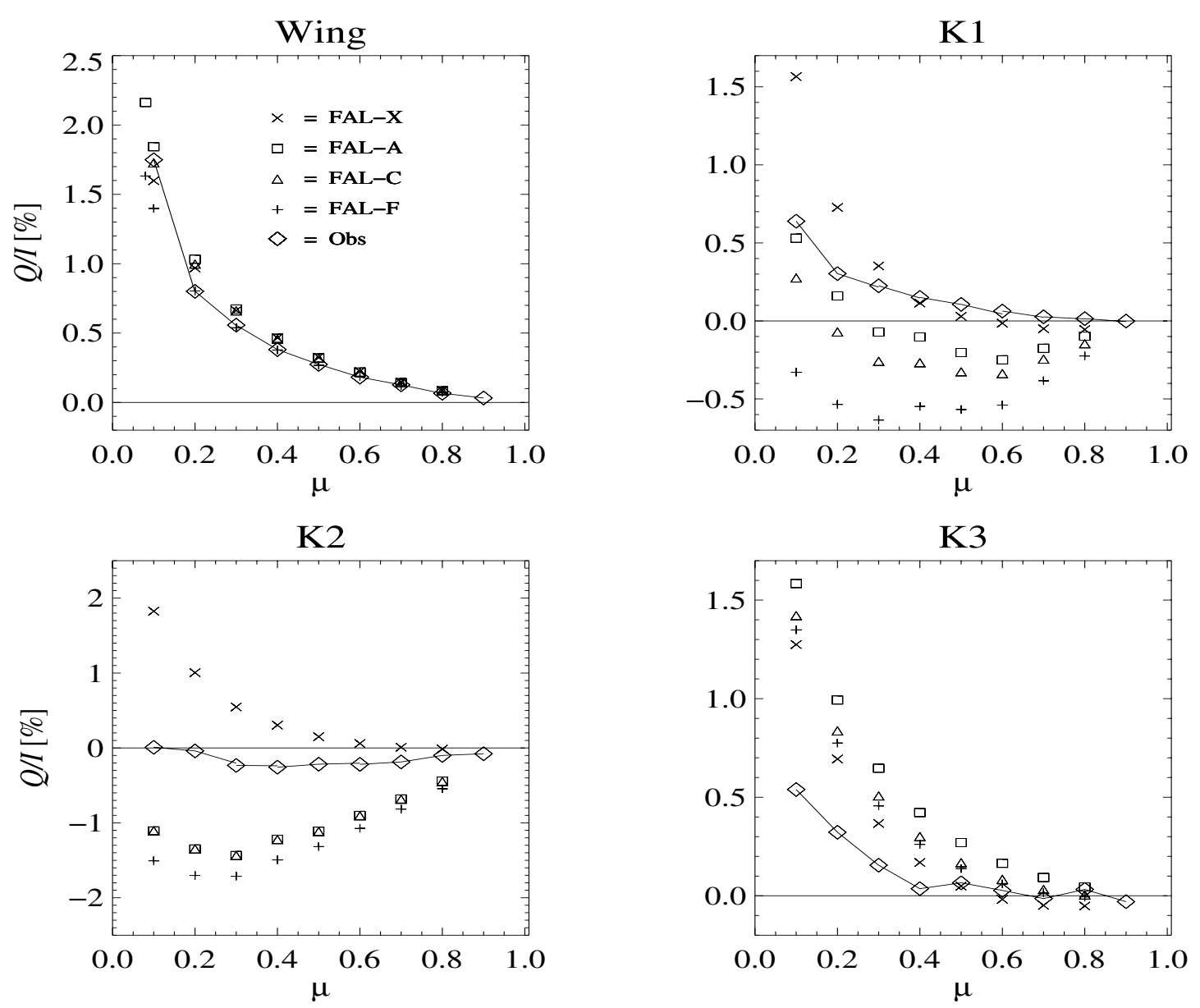

Fig. 1. Observed and calculated polarization signals as functions of heliocentric angle $\mu$ (in steps of 0.1 ) at four distinct wavelength positions. The spectra are calculated for standard models FAL-A, FAL-C, FAL-F and FAL-X. The sizes of the symbols for the observations approximately represent the uncertainties in the heliocentric angle $(\Delta \mu \approx 0.02)$ and in the zero point of the polarization scale $(\Delta p \approx 0.02 \%)$. To indicate the strong dependence of the polarization signal on $\mu$ at the very limb, the upper left panel contains the additional angle $\mu=0.08$ (models FAL-A and FAL-F only). The symbols representing the observations are interconnected for better visibility.

\section{Angular dependence of the polarization profiles}

To get an impression of the behavior of the polarized spectra as a function of the heliocentric angle, we select four outstanding wavelength positions in the $Q / I$ spectrum. These positions correspond to the maximum polarization in the wing, the second weak maximum in the inner wing, the minimum polarization close to the core and the line core. Because these positions almost coincide with the well-known extremal positions in the intensity spectrum (see the vertical lines in Fig. 4) we call them wing, $\mathrm{K} 1$, K2 and $\mathrm{K} 3$ thereafter. For the polarized spectrum the slightly $\mu$-dependent positions are indicated in Fig. 2. In Fig. 1 the polarization at these wavelength positions is plotted against the heliocentric position $\mu$ (the cosine of the heliocentric angle) for four model atmospheres and the observations. The size of the symbols for the observations (diamonds) indicates the approximate errors in $\mu$ (determined during the observation) and in the absolute zero point of polarization (determined near strong blend lines). Note that due to the geometry of the scattering process, the amount of polarization decreases towards the disk center.

\subsection{Wing maximum}

The calculations for the wing - which is formed in the midphotosphere (200-300 km above $\tau=0$ ) - almost perfectly reproduce the observations. None of the models may be ruled out or favored. Although it seems that the FAL-F model gives slightly better results, one has to remember the strong dependence of the wing polarization on the elastic collision rate. The calculations are performed with the same collision rate as determined in Paper Ib (i.e. $\gamma_{\mathrm{VW}}=1.8 \times 10^{-8} \mathrm{~cm}^{3} \mathrm{~s}^{-1}$ ). A change of only $20 \%$ in this rate results in a (relative) change of approximately $10 \%$ in the resulting polarization. At wavelengths near the core ( $\pm 3-5 \AA)$ collisions may be neglected because of the low density at the respective formation height.

The observational error in the determination of the heliocentric angle $(\Delta \mu \approx 0.02)$ has to be considered as well, especially for small $\mu$. The observation at $\mu=0.1$ shows a wing polarization of $1.8 \%$, whereas Gandorfer (2002), at the same nominal $\mu$ value, measures only $1.3 \%$ (in Paper Ib we estimated the cosine of the heliocentric angle for the latter observation to be 0.12 ). Our observed data point at nominally $\mu=0.1$ seems to be consistent with a value of $\mu \approx 0.08-0.09$. For this reason, we added the values for two model atmospheres at $\mu=0.08$.

\section{2. $K 1$}

At the K1 wavelength - formed at 400-750 $\mathrm{km}$ above $\tau=0-$, all the models with a hot chromosphere show the same behavior but with different strength: the temperature rise produces negative contributions to the polarization. This is in contradiction with 
the observations, especially near the disk center. The cold model FAL-X shows qualitatively the correct behavior but is not able to reproduce the proper $\mu$ dependency either: at the limb, this model gives much more polarization, while near disk center it produces less polarization than observed. The effect seems to be caused by the fact that in the lower part of the $\mathrm{K} 1$ formation range (i.e. at $400-500 \mathrm{~km}$ ), which is seen at $\mu \geq 0.5$, the FAL-X model has a less steep temperature curve. At the limb, the same part of the atmosphere is seen in the near wing close to the polarization minimum at $3932 \AA$, where the calculated spectrum for FAL-X indeed shows a very low polarization signal (see also Paper Ib).

In the upper part of the $\mathrm{K} 1$ formation range (i.e. at small $\mu$ ), the observations show less polarization than the FAL-X model. This seems to be an indication of the enhanced influence of hotter components.

\section{3. $K 2$}

While modeling and observations at $\mathrm{K} 1$ diverge in different ways for different heliocentric angles, the situation at the $\mathrm{K} 2$ wavelength (formation height: $500-1500 \mathrm{~km}$ ) is much more uniform: the three hot models show only minor variations as noted already by Saliba (1985). The observational data are located in between the hot and cold models, with a tendency towards the cold model at disk center and towards the hot models at the limb. Because smaller $\mu$ sample higher regions in the atmosphere, this trend may be due to the increasing influence of hot components at larger heights. Although we cannot exclude some influence of Hanle depolarization at K2, the calculated spectra fit the observations only when we mix cold and hot components.

\subsection{Line core (K3)}

The situation in the line core (formation height: $\geq 1600 \mathrm{~km}$ ) is completely different and may be understood in terms of magnetic field effects. All modeled spectra have been computed without Hanle depolarization, i.e. for $B=0$. The observations always show less absolute polarization (even when the polarization is negative). This is exactly the signature one would expect from depolarization via the Hanle effect. The magnetically influenced parts of the observations will be discussed in a forthcoming paper.

In general, Fig. 1 confirms that none of our standard set of models is able to explain the observations. Our bifurcated chromosphere used in Paper Ib is strongly confirmed. The only part of the line spectrum where a single model fits the observed profiles is the extended wing, which is formed in the mid-photosphere much below the classical temperature minimum and where variations in the mean temperature are much smaller, as evidences by the very small observed spatial variation (along the slit) in the wings (not exceeding the noise level).

\section{Comparison of observations with two-component mixture}

Figure 2 shows observations and model calculations as performed in Paper Ib for three representative center-to-limb distances. The thin dashed lines represent the individual solutions for one single model atmosphere, the thick solid line the mixture with the mixing ratio (FAL-C/FAL-X:0.55/0.45). As discussed in Sect. 2 we find no agreement between observations and the individual models at wavelengths around $\mathrm{K} 1$ and $\mathrm{K} 2$. On the other

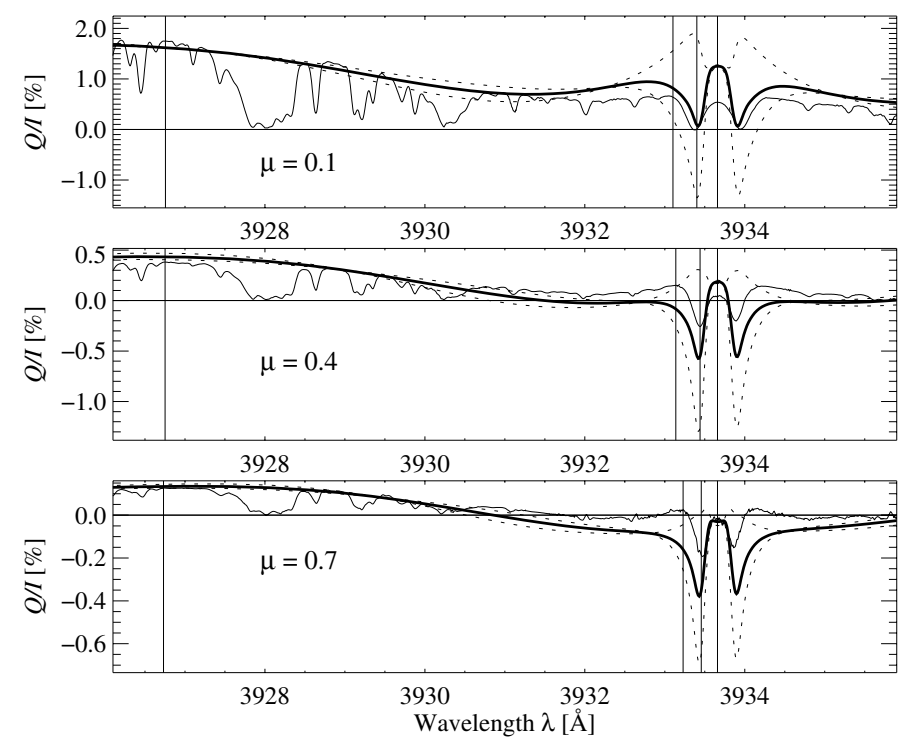

Fig. 2. Comparison of observations (thin solid lines) and calculated spectra at three selected center-to-limb distances $\mu$. The computed spectra represent the same atmospheric models as used in Paper Ib: FAL-C (lower thin dashed curves) and FAL-X (upper thin dashed curves). The mixed spectrum (thick solid curves) is obtained with the same mixing ratio (FAL-C/FAL-X:0.55/0.45) as in Paper Ib. The vertical lines indicate the four selected wavelength positions we use in Fig. 1.

hand, the mixture agrees better although a systematic shift from smaller to larger $\mu$ may be seen.

The measurements near the disk center show surprisingly large polarization at $\mathrm{K} 1$. It is not possible to fit the spectra at $\mathrm{K} 1$ for larger $\mu$ with a mixture, because none of the atmospheres produces enough polarization to reproduce the observations. We therefore conclude that the atmospheres have to be adjusted at the formation height of the K1 polarization (i.e. near the classical temperature minimum). To enhance the calculated polarization, at least one of the atmospheric components needs a steeper temperature gradient and therefore a lower temperature minimum. A quantitative approach is given in the next section, where we search for single atmosphere solutions for the individual observations.

Furthermore, an attempt to change the global mixing ratio does not help, because an increase of polarization at larger $\mu$ (by increasing the FAL-X contribution) leads to an unwanted increase at small $\mu$. The same effect also occurs when changing the temperature curve of the individual atmospheres. We conclude that the constraint that is imposed by the observations at different angles is very tight, because the physical state at a certain height in the solar atmosphere affects all $\mu$ positions but at different wavelengths. When the atmosphere is changed at a given height, the change is reflected in all spectra in a similar way. This leads to the conclusion that perfect agreement between observations and calculated spectra for all heliocentric angles is not possible with a height-independent mixing ratio of two atmospheres that are similar to the standard atmospheres used here. As mentioned in the previous section, the results are consistent with a situation where the hotter components have increased influence in the higher atmosphere. This fits well with the current picture of hot magnetic canopies spreading out above the photosphere. 

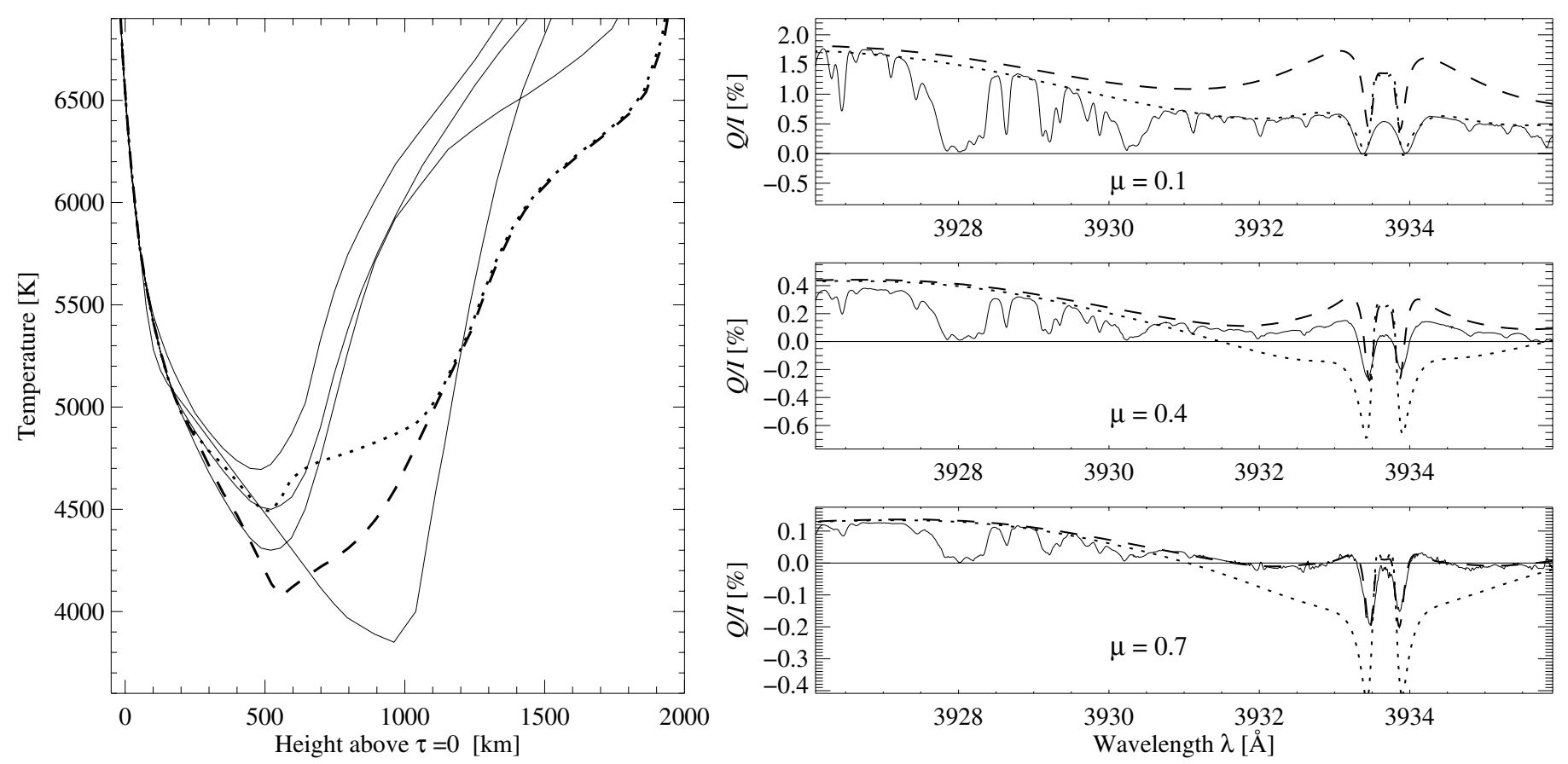

Fig. 3. Left panel: temperature curve of the optimum solutions for $\mu=0.1$ (dotted) and $\mu=0.7$ (dashed). The four standard models used in this work are drawn for reference (thin solid). Right panel: optimum solutions for $\mu=0.1$ (dotted) and $\mu=0.7$ (dashed) versus observations at three distinct heliocentric angles. A single atmosphere is not able to fit observations at all angles. A change of the temperature curve of one atmosphere has influence on every angle. Note that the systematic shift with increasing $\mu$ is consistent with the one in Fig. 2. A fit of the spectrum at a large $\mu$ needs a much colder model.

\section{Single atmosphere fit}

Starting from a standard model, we generate a new selfconsistent model atmosphere with a given temperature curve. The new model is then used to calculate the polarized spectrum. From the difference between observations and the calculated spectrum at a given heliocentric angle, we derive an improved temperature curve, always following the idea that a steeper (positive) gradient produces more polarization. We start the inversion process in the lower atmosphere (i.e. in the wings) and then continuously extend the optimization to higher layers up to $1500 \mathrm{~km}$ above $\tau=0$. The optimum solutions for two angles are shown in the left panel of Fig. 3 as dashed $(\mu=0.7)$ and dotted $(\mu=$ 0.1 ) curves. The four standard models (FAL-A, FAL-C, FAL-F, FAL-X) are shown as solid lines. In the right panel of Fig. 3 the calculated spectra are plotted together with the observed profiles for the same angles as in the previous figures.

The circumstance that already minor changes in the temperature curve significantly change the amount of polarization, and that the calculation of many variations around the optimum solution give no improvement, suggests that the optimized solutions are unique except above $1200 \mathrm{~km}$ because of the rapidly decreasing influence of the temperature curve. Therefore an arbitrary temperature curve has been chosen for the higher part of the chromosphere.

One should mention that only one given $\mu$ position can be optimized with one atmosphere. The two solutions for $\mu=0.1$ and $\mu=0.7$ differ strongly in their temperature curves.

It was foreseeable from the considerations in Sects. 3 and 4 that the optimum fit for $\mu=0.7$ needs a steeper temperature gradient in the photosphere. Interestingly, the temperature curve is almost identical to the FAL-A model but with a deeper temperature minimum at a higher location. The location of the temperature minimum can be accurately determined, because even a small change of its height results in a strong wavelength shift of the polarization decline near K1. The depth of the polarization dip at $\mathrm{K} 2$ is sensitive to the temperature increase in the low chromosphere. There the optimum solution has only a gently increasing temperature - in strong contrast to the standard models.

Near the limb $(\mu=0.1)$ the optimum solution in the photosphere is very similar to the FAL-C model. To prevent the strong dip at K2, the temperature may only increase slowly in the lower chromosphere. To fit the polarization from K1 towards $\mathrm{K} 2$, one needs an abrupt increase in temperature above the photosphere. At $\mu=0.1$ the optimum solution has a temperature jump of almost $300 \mathrm{~K}$ to fit the spectra on the red side of the line center. On the blue side an even larger jump would be necessary. Note that if the steep increase of the temperature would continue in the lower chromosphere $(700-1200 \mathrm{~km})$, then the polarization spectrum would coincide with that of the FAL-C model, i.e. the dip would be too deep and the local polarization maximum at K1 would disappear.

In Fig. 4 the intensity spectrum of the observations and the two single atmosphere solutions are plotted for the same heliocentric angles as in Fig. 3. Since the continuum level in the observed intensity is not known, we have scaled the observed intensity to fit the calculated profiles in the wings. None of the two solutions shows the characteristic intensity inversion observed at K2. Therefore the optimum solutions for the polarized spectra are not hot enough to produce $\mathrm{K} 2$ emission. The conclusion is that a single atmosphere solution is unable to fit both intensity and polarization even at one single heliocentric angle.

In summary we find that the single atmosphere solutions seem to favor models with a lower $T_{\min }$ positioned slightly above the standard height of the temperature minimum and with a step-like increase of the temperature followed by a slow increase throughout the lower chromosphere. However, these solutions cannot reproduce more than the polarized spectrum at one 


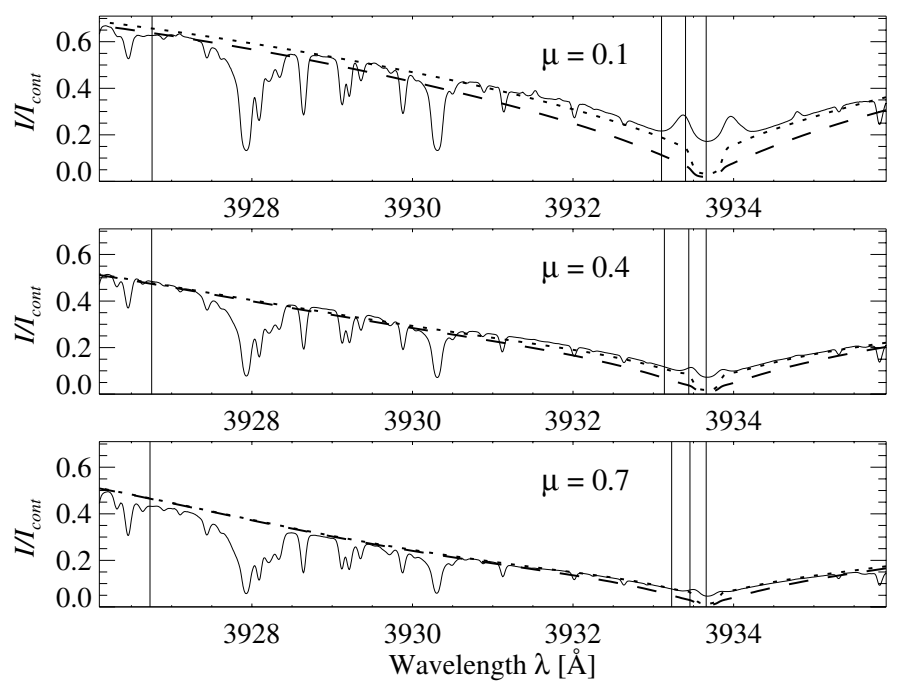

Fig. 4. Intensity spectra associated with the two optimum solutions for the polarized spectra (same line styles as in Fig. 3). The unknown continuum level of the observations has been scaled to fit the calculations. None of the synthetic spectra shows the observed intensity reversal at $\mathrm{K} 1 / \mathrm{K} 2$, which is produced by the chromospheric temperature rise. Therefore the models are too cold to explain the intensity. The vertical lines indicate the selected wavelength positions as used in the text.

specific angle. For a fit to the intensity profile one needs at least a small hot component that contributes non-linearly to the intensity increase at $\mathrm{K} 2$. When using hotter components, the cold component has to be even colder than the optimum solutions found here.

If we assume that the atmospheric conditions at different heliocentric positions are basically the same and that the cold component at $\mu=0.1$ therefore has to be at least as cold as the optimum solution for $\mu=0.7$ (it actually has to be colder, because hotter components are needed to fit the intensity spectrum at $\mu=0.7$ ), we end up with a multi-component picture of the quiet Sun's atmosphere, where the cold components dominate, but with increasing contributions from hot components at larger heights. The two optimum solutions for large and small $\mu$ values start to differ already in the mid-photosphere, which leads to the conclusion that the hot components already exist at these heights.

\section{Influence of temperature and turbulence}

\subsection{Influence of temperature variations}

To get a quantitative understanding of the temperature sensitivity of the linear polarization, we have varied the temperature curve of the standard FAL-C model. The temperature gradient is changed between the continuum formation level $(T \approx 6450 \mathrm{~K})$ and the mid-chromosphere around $1400 \mathrm{~km}$ above the $\tau=0$ level, where the same temperature prevails. A self-consistent atmosphere is generated with the temperature gradient changed by $\pm 10 \%$ for all heights in the selected height range. This causes the temperature minimum in these models to be increased or lowered by slightly more than $200 \mathrm{~K}$. The left part of Fig. 5 shows the resulting polarization profiles. The amount of polarization in the wings is changed by almost $20 \%$, which demonstrates the high sensitivity to the shape of the temperature curve. The relative polarization change over the whole wing region is almost constant up to the wavelength where the polarization crosses the zero line (3933 $\AA$ ). There positive contributions from the lower
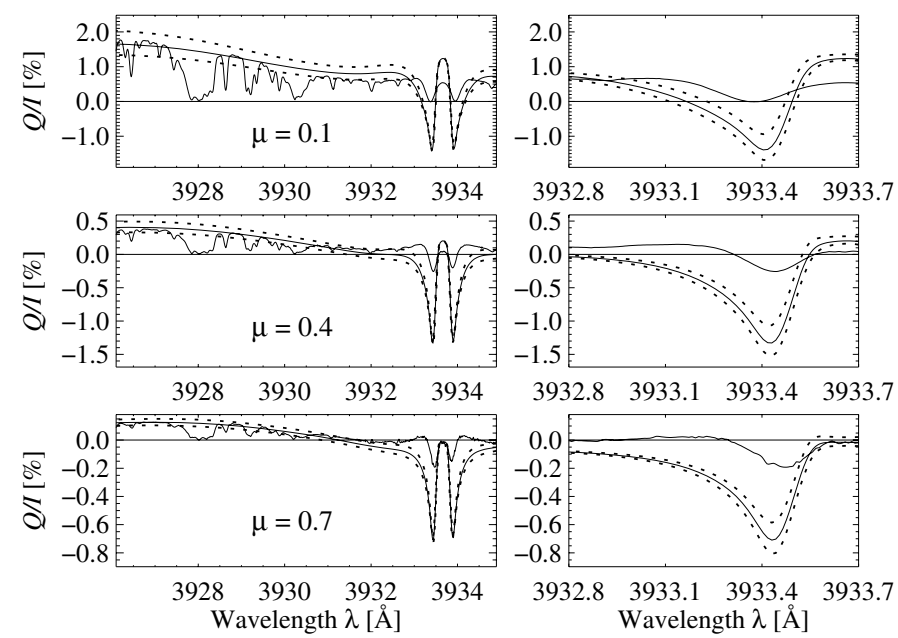

Fig. 5. Synthetic polarization spectra obtained after changing the temperature gradient of the original FAL-C model for the same three heliocentric positions $\mu$ as in the previous figures. Left panel: spectra for the FAL-C model (solid) and two models with $\delta(\nabla T)= \pm 10 \%$ (dotted) from the photosphere to the lower chromosphere. Right panel: FAL-C model (solid) and two models with $\delta(\nabla T)= \pm 20 \%$ (dotted) above the temperature minimum, illustrating the strong influence of the chromospheric temperature rise on the polarized spectrum at $\mathrm{K} 1$ and $\mathrm{K} 2$.

layers and negative contributions from the temperature rise in the upper layers of the atmosphere cancel each other. As can be seen from Fig. 5, the polarization near K2 is not changed so much because of this cancellation and because the temperature sensitivity decreases with increasing height.

To isolate the effect of the temperature rise we calculate the spectra for two atmospheres, in which the temperature gradient is changed only between the temperature minimum and the top of the atmosphere. The right panel of Fig. 5 shows the polarization profile produced by the FAL-C model and two models where $\nabla T$ has been changed by $20 \%$. As one may see, the influence of the chromospheric temperature rise starts near K1 (in the case of $\mu=0.1$ even further in the wings) and is almost as strong as that of the photospheric temperature curve. This is consistent with the results from Sect. 5, where the temperature rise for the single atmosphere solutions had to be lowered drastically to reproduce the weak dip at K2 in the observations.

\subsection{Influence of turbulence}

Figure 6 shows the effects of variations of the micro-turbulence in the upper parts of the FAL-C model. The influence of microturbulence in the photosphere can be neglected. No effect on the linear polarization can be seen in the wings. However, near $\mathrm{K} 2$ and the line center, which originate in the higher parts of the atmosphere, turbulence may not be disregarded. Above selected heights in the atmosphere, we increase the standard micro-turbulence of the FAL-C model by $50 \%$. The thick solid line represents an atmosphere in which $v_{\text {mic }}$ has been increased for $z \geq 1800 \mathrm{~km}$. At $\mu=0.7$ there is almost no difference with respect to the original FAL-C (thin solid, mostly hidden). At $\mu=0.1$, on the other hand, the two spectra may easily be distinguished because the enhanced turbulence causes a larger width of the line core polarization. The thick dotted line (enlarged $v_{\text {mic }}$ for $z \geq 1500 \mathrm{~km}$ ) behaves the sameway, but with a more affected minimum, which is now shifted towards the wing. The polarization at the minimum is also raised slightly, although not enough to reproduce the observations. For the two additional 


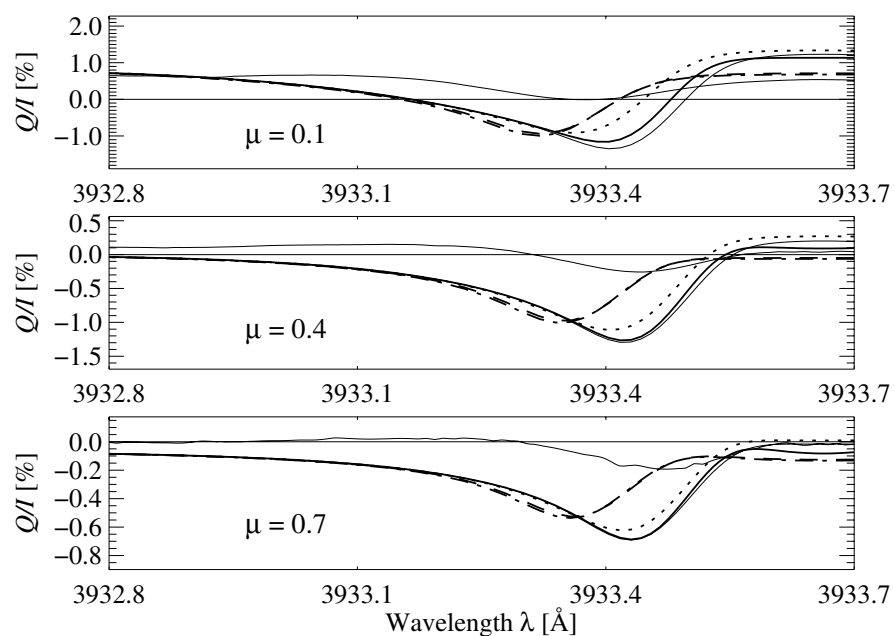

Fig. 6. Influence of micro-turbulence variations on the FAL-C model spectra. The profiles result from models with increased $v_{\text {mic }}$ (by a factor of 1.5). The difference between the models is the heights in the atmosphere where the micro-turbulence is increased: original FAL-C (lower thin solid, almost hidden), enlarged $v_{\text {mic }}$ for $z \geq 1800 \mathrm{~km}$ (thick solid), enlarged $v_{\text {mic }}$ for $z \geq 1500 \mathrm{~km}$ (thick dotted), enlarged $v_{\text {mic }}$ for $z \geq 800 \mathrm{~km}$ (thick dashed) and for $z \geq 400 \mathrm{~km}$ (thick dash-dotted, almost hidden by the dashed line). The upper thin solid lines represent the observed profiles.

atmospheres with $v_{\text {mic }}$ enhanced for $z \geq 800 \mathrm{~km}$ (dashed) and for $z \geq 400 \mathrm{~km}$ (dash-dotted) the situation is analogous. The minimum is shifted much too much towards the wing and is still far below the observations. A raise of $50 \%$ in $v_{\text {mic }}$ is a drastic but not totally unrealistic change. We conclude that while the K2 polarization minimum is influenced by turbulence, the original turbulence of the FAL models leads to quite good agreement with the observations when considering the wavelength position of the $\mathrm{K} 2$ polarization minimum. On the other hand, micro-turbulence may not be used to explain the weakness of the observed polarization dip near $\mathrm{K} 2$.

\section{Conclusions}

We used full Stokes vector spectra obtained with the UV-version of ZIMPOL to investigate the center-to-limb behavior of the linear polarization of the Ca II K line. As shown in our previous work (Paper Ib) the Ca II $\mathrm{K}$ scattering polarization is a good tool to diagnose the temperature structure of the high photosphere and the low chromosphere. We compared the observed polarization and its center-to-limb variation to synthetic spectra calculated on the basis of 1D standard atmospheric models. While the observed wing polarization originating from the high photosphere is in good agreement with calculations for all heliocentric positions, no agreement is found for the polarization around $\mathrm{K} 1$ and $\mathrm{K} 2$.

At K1 and K2 the observed polarization lies between the calculated spectra for atmospheric models with and without temperature rise for all heliocentric positions, which is in accordance with the results found in Paper Ib for $\mu=0.1$. Also it is possible to achieve a qualitative agreement when a two-component model with about equal fractions of cold and hot components is used. However, a small systematic shift cannot be eliminated with a constant mixing of two components. Towards disk center, colder atmospheric components are needed to explain observations. The observations near the limb on the other hand, originating from slightly higher layers, show an increased influence of the hotter components. This effect starts already in the mid-photosphere.

We also determined the quantitative influence of the temperature curve on the polarization profile, which is very strong in the photosphere and in the low chromosphere. A variation of the temperature minimum of $100 \mathrm{~K}$ (corresponding to a $5 \%$ change of the temperature gradient throughout the photosphere) results in a change of the wing polarization of approximately $10 \%$. This confirms the high sensitivity of scattering polarization to atmospheric conditions. Modifications of the micro-turbulence show that its influence is weak and relevant only near the line core. Mainly the position of the K2 polarization minimum is influenced by micro-turbulence. The comparison of observations with calculations shows that in the standard models, microturbulence is overestimated in the lowest chromosphere while underestimated in the upper chromosphere.

We searched for single atmosphere solutions to fit the observed polarization profile. Though it is possible to find a solution for the polarized spectrum at every single heliocentric position, it is impossible to fit observations at all positions simultaneously. Furthermore, these solutions cannot reproduce the observed intensity profile. They do not show the typical intensity reversal caused by the temperature rise in the chromosphere. We conclude that a static one-dimensional single atmosphere solution is in no way able to explain observations and that a more realistic model must contain substantial parts of a very cold atmosphere (minimum below $4000 \mathrm{~K}$, less steep or no temperature rise) and also parts of a very hot chromosphere (to reproduce the intensity reversal).

Even multi-component models consisting of 1D atmospheric models for each component are found to be unable to provide detailed fits to the observations for all center-to-limb distances simultaneously. In such idealized models the hot and cold components are assumed to be optically independent of each other, which is certainly not the case in realistic geometries with narrow flux tubes that rapidly expand with height. This indicates that multi-dimensional radiative transfer would be needed for a fully self-consistent treatment.

Previous treatments of the chromospheric structure with 3D geometries based on MHD simulations, like those of Skartlien et al. (2000) and Wedemeyer et al. (2004), are of no use here, since they are based on strongly simplified unpolarized radiative transfer. This also applies to the unpolarized 1.5D modeling of 2D expanding flux tubes by Solanki et al. (1991). More relevant to our case is the self-consistent modeling of polarized scattering in photospheric lines ( $\mathrm{Sr}$ I and molecular lines) in 3D geometries obtained from MHD simulations by Trujillo Bueno et al. (2004). However, it uses the simplifying assumption of complete redistribution, which is appropriate for photospheric lines but totally inadequate for strong chromospheric lines like the Ca II K line. For such lines the inclusion of partial frequency redistribution (PRD) is a must.

Recently the general theory for PRD of scattering polarization in magnetized media has been given a comprehensive formulation in a series of papers by Sampoorna et al. (2007a,b), but a general computer code for polarized PRD is not yet available, and such computations are extremely demanding in terms of computing resources, even without multi-dimensional geometry. Therefore the fully self-consistent application of this approach is still some time away in the future, and various idealizations will still be needed before a true multi-dimensional treatment is possible. 
Our next step will therefore not be multi-dimensional radiative transfer, but the empirical exploration of the spatial structuring of the $\mathrm{Ca} \mathrm{K}$ scattering polarization. In the present paper we have focused on the spatially averaged properties of the scattering polarization. However, all our recorded Stokes spectra show significant fluctuations of the polarization, in particular in the line core, where the Hanle effect is active. Magnetic fields are omnipresent, also in the most quiet solar regions. The determined empirical properties of these magnetically - induced fluctuations will guide us to the next level of physical understanding of the Ca K scattering polarization. This is the topic of the next paper in the present series.

Acknowledgements. We are indebted to Han Uitenbroek who provided us with his wonderful RH code for Non-LTE radiative transfer, which we have used to compute Stokes I. D. Fluri has programmed the polarized part of our radiative transfer code. A. Feller, C. Thalmann, D. Gisler, M. Bianda, R. Ramelli, A. Gandorfer, and C. Keller helped to obtain the outstanding observational data used in this work. R.H. appreciates the flexibility of Prof. Dr. Norbert Dillier concerning the working hours at the University Hospital of Zurich. We thank the referee for his constructive suggestions which helped us to improve our work.

\section{References}

Auer, L. H., Rees, D. E., \& Stenflo, J. O. 1980, 88, 302

Avrett, E. H. 1995, in Infrared tools for Solar Astrophysics: What's Next, ed.

J. R. Kuhn, \& M. J. Penn, NSO/SP Summer Workshop, NSO, Sunspot, 303 Fluri, D. M., \& Stenflo, J. O. 2003, 398, 763

Fontenla, J. M., Avrett, E. H., \& Loeser, R. 1993, 406, 319

Gandorfer, A. 2002, The Second Solar Spectrum, Vol II: $3910 \AA$ to $4630 \AA$ (Zurich: $\mathrm{VdF}$ )

Gandorfer, A. M., Povel, H. P., Steiner, P., et al. 2004, 422, 703

Holzreuter, R. 2003, in Solar Polarization, Proc. 3rd SPW, ed. J. Trujillo Bueno, \& J. Sánchez Almeida, ASP Conf. Ser.

Holzreuter, R., Fluri, D. M., \& Stenflo, J. O. 2005, 434, 713

Holzreuter, R., Fluri, D. M., \& Stenflo, J. O. 2006, 449, L41

Povel, H. P. 1995, 34, 1870

Saliba, G. J. 1985, 98, 1

Sampoorna, M., Nagendra, K. N., \& Stenflo, J. O. 2007a, submitted

Sampoorna, M., Nagendra, K. N., \& Stenflo, J. O. 2007b, submitted

Skartlien, R., Stein, R. F., \& Nordlund, A. 2000, 541, 468

Solanki, S. K., Steiner, O., \& Uitenbroek, H. 1991, 250, 220

Stenflo, J. O. 1980, 84, 68

Stenflo, J. O. 2006, in Solar Polarization, Proc. 4th SPW, ed. R. Casini, \& B. W. Lites, ASP Conf. Ser., 358, 215

Stenflo, J. O., Gandorfer, A., Holzreuter, R., et al. 2002, 389, 314

Stenflo, J. O., Gandorfer, A., Wenzler, T., \& Keller, C. U. 2001, 367, 1033

Stenflo, J. O., \& Keller, C. U. 1997, 321, 927

Stenflo, J. O., Twerenbold, D., \& Harvey, J. W. 1983, 52, 161

Trujillo Bueno, J., Shchukina, N., \& Asensio Ramos, A. 2004, 430, 326

Wedemeyer, S., Freytag, B., Steffen, M., Ludwig, H.-G., \& Holweger, H. 2004, A\&A, 414, 1121 